\title{
Functional Gastrointestinal Disorders Diagnosed by Rome III Questionnaire in Korea
}

\author{
Jae Myung Park, Myung-Gyu Choi,* Yu Kyung Cho, In Seok Lee, Jin II Kim, Sang Woo Kim and In-Sik Chung \\ Division of Gastroenterology, Department of Internal Medicine, College of Medicine, The Catholic University of Korea, Seoul, Korea
}

\begin{abstract}
Background/Aims
Rome criteria classifying functional gastrointestinal disorder (FGID) were updated. The aims of this study were to assess the spectra of FGID and to evaluate the applicability of Rome III criteria in Korea.

\section{Methods}

New patients who visited 2 primary clinics and 2 tertiary care hospitals were consecutively invited to complete questionnaires. These consisted of questionnaires for FGID based on Rome III criteria and symptom checklist-90-revised for somatization, depression and anxiety.

\section{Results}

A total of 786 patients was participated. Among them, FGID was observed in $49.7 \%$. In the patients with FGID, functional dyspepsia was most common (46.0\%) followed by irritable bowel syndrome (IBS, 40.2\%). Functional dyspepsia was most common both in the primary care clinics and tertiary care hospitals. Postprandial distress syndrome was the most common subtype and the frequency of epigastric pain syndrome was low. There were few responders for constipation as Bristol types 1 and 2 and for diarrhea as types 6 and 7 in subtype classification using the Bristol Stool Form Scale. Thereby, unspecified IBS defined by stool form was unexpectedly common in $43.9 \%$ of IBS, whereas unspedified IBS defined by Rome III definition was $5.1 \%$. Patients with overlap FGIDs had higher score of anxiety, depression or somatization.
\end{abstract}

\section{Conclusions}

FGIDs were common both in primary care clinics and tertiary care hospitals of Korea. Overlap FGIDs is still common by Rome III criteria, which may limits its wide application.

(J Neurogastroenterol Motil 2011;17:279-286)

\section{Key Words}

Classification; Dyspepsia; Gastrointestinal diseases; Irritable bowel syndrome

\section{Introduction}

Rome criteria were introduced for a standard to diagnose and classify the functional gastrointestinal disorder (FGID) in the early 1990's. Along with many studies and publications on the FGIDs, the criteria were then updated as Rome II in 2000 and Rome III in 2006. ${ }^{1,2}$ Compared with Rome II criteria, there were major changes in Rome III version as follows: changes in chronological criteria for diagnosis of FGIDs from 12 months to 6

Received: December 23, 2010 Revised: May 8, 2011 Accepted: May 11, 2011

(c) This is an Open Access article distributed under the terms of the Creative Commons Attribution Non-Commercial License (http://creativecommons. org/licenses/by-nc/3.0) which permits unrestricted non-commercial use, distribution, and reproduction in any medium, provided the original work is properly cited.

*Correspondence: Myung-Gyu Choi, MD Division of Gastroenterology, Department of Internal Medicine, College of Medicine, The Catholic University of Korea, 505 Banpo-dong, Seocho-gu, Seoul 137-701, Korea

Financial support: None.

Tel: +82-2-2258-6017, Fax: +82-2-2258-2055, E-mail: choim@catholic.ac.kr

Conflicts of interest: None. 
months for the onset, and from 6 months to 3 months for the activity of the symptoms; changes of subtypes in functional dyspepsia (FD) as postprandial distress syndrome (PDS) and epigastric pain syndromes (EPS); more strict criteria for functional disorders of the gallbladder and sphincter of Oddi; and revision in subtyping irritable bowel syndrome (IBS) using stool consistency.

However, Rome III has not been fully validated in that many of the FGIDs are underrepresented in the validation samples of FGID patients. Rome III criteria have not been considered for the differences in the prevalence of organic diseases and medical services in different geographic regions. The next version of Rome criteria is expected to be developed inevitably based on the researches using Rome III criteria, as experienced in Rome II version.

The aims of this study were to assess the spectra of FGIDs and to study the local applicability of Rome III criteria in Korea. Specifically, the present study evaluated the pattern of FGIDs in primary care clinics and tertiary care hospitals and assessed problems in diagnosing the FGIDs by Rome III criteria. Our study also investigated the psychological problems in the patients with FGIDs,

\section{Materials and Methods}

\section{Study Subjects}

This study involved 2 primary clinics and 2 tertiary care hospitals. Between April and September of 2007, we enrolled all consecutive new patients with chronic gastrointestinal symptoms. We excluded 193 patients whose symptoms were less than 6 months; 65 patients from primary clinics and 128 patients from tertiary hospitals. All patients were asked to complete questionnaires, examined physically and evaluated with complete blood counts, biochemical and urinalysis tests. All patients underwent esophago-gastro-duodenoscopy to exclude organic diseases. If clinically indicated, thyroid function tests, abdominal ultrasonography and total colonoscopy was performed. Patients who took medication specific for gastrointestinal system for 6 months or less before this study were also excluded. After the clinical evaluations patients with abnormal laboratory tests, endoscopic examination and abdominal ultrasonography were also excluded from this study. Patients who did not complete examinations or questionnaires were also excluded from final analysis. The study was approved by the institutional review board of Seoul St.
Mary's Hospital.

\section{Procedures}

Demographic data, body weight and height were examined from all patients. Rome III questionnaire was used to evaluate the spectra of FGID. To evaluate the status of somatization, anxiety and depression, eligible patients were also asked to complete the symptom checklist-90-revised (SCL-90R).

\section{Questionnaire}

The questionnaires consisted of Rome III questions and dimensions of anxiety, depression and somatization from SCL-90R.

\section{Rome III questionnaire}

We adapted Rome III questionnaire into Korean through a process of translation and back-translation before this study. The translation process was as follows. Two professional translators, who were native speakers of Korean and fluent in English, undertook 2 translations independently. Based on these translations, and on consultation with a specialist in Korean, a reconciled version of the questionnaire was developed. A report was then prepared and reviewed. A native speaker of English, also fluent in Korean, then translated the Korean version back into English. The original and back-translated (English) versions were compared to detect any misunderstandings, mistranslations, or inaccuracies in the Korean draft questionnaire. All decisions on the use of Korean expressions were carefully reviewed to ensure that the sense of questionnaire items was retained. A second Korean language version of the Rome III questionnaire was then prepared, and feedback was obtained from experts in gastroenterology. The translated Rome III questionnaire was administered to 10 patients with FGIDs to assess the clarity, understandability, cultural relevance and appropriateness of the translation's wording. Based on the respondents' feedback, the final Korean version of the Rome III questionnaire was produced. Final Korean version of Rome III questionnaire consisted of 74 questions. We did not include the original questions which were asked to rule out organic diseases and not used in scoring algorithm. We also asked the Bristol types with representative stool form figures in 1 question. Patients recalled the frequency of stool form during the recent 3-month period. The response scale of Bristol Stool Form was as follow; never, about 25\%, 50\%, 75\% and $100 \%$ of the time. During the validation study, it took 10 to 30 minutes to complete the questionnaire.

To diagnose FD, patients must have one or more of following symptoms for at least 3 months with symptom onset at least 6 
months previously: bothersome postprandial fullness, early satiation, epigastric pain, epigastric burning with no evidence of structural disease, including the use of upper endoscopy, which is likely to explain the symptoms. FD was classified into PDS and EPS subtypes.

Diagnosis of IBS was based on the presence of abdominal pain or discomfort for at least 3 months in the previous 6 months, with 2 or more of the following symptoms: pain improved after defecation, symptoms associated with a change in frequency of stool and symptoms associated with a change in the form of stool. We subtyped IBS by using questions in a text form on hard or lumpy stools and loose or watery stools. The response scale of these questions were as follow; never, about 25\%, 50\%, 75\% and $100 \%$ of the time. We intended to validate these questions by using a pictorial form which could enhance patients' understanding. Patients were asked to fill the subjective question on the percentage of stool form during the time.

\section{Symptom checklist-90-revised}

The SCL-90R is a self-report questionnaire with 90 queries exploring symptom dimensions of disorders from anxiety and depression to psychoticism and paranoid ideation. ${ }^{3}$ The effectiveness of the SCL-90R as a screening instrument has been validated in Korea. ${ }^{4}$ Thirty-five of 90 queries, containing the dimension of anxiety, depression or somatization, were asked to be completed. In the SCL-90R, participants responded on a 5-point Likert scale and graded on a 0-4 score, with higher scores indicating more distress. On each scale of the SCL-90-R, results

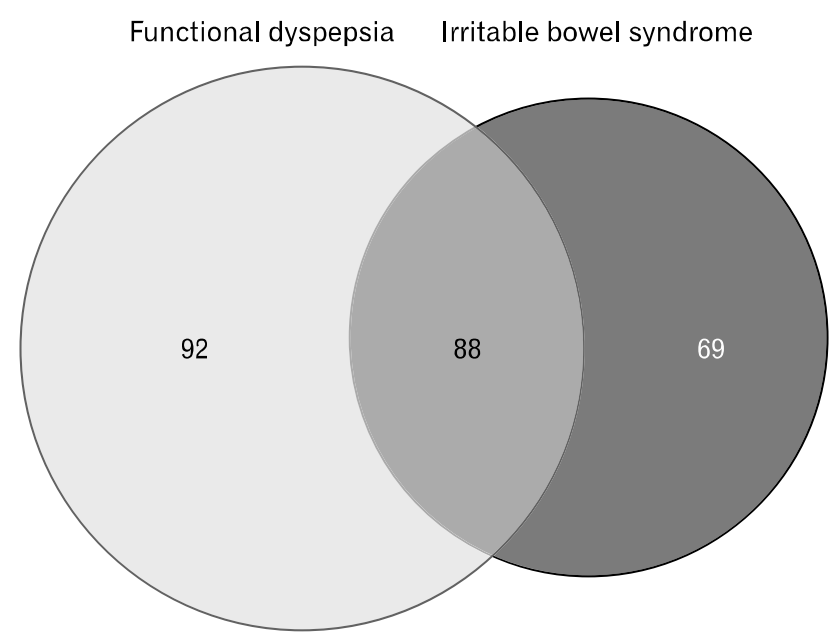

Figure. Overlap between irritable bowel syndrome (IBS) and functional dyspepsia (FD). Eighty eight of the $180 \mathrm{FD}$ patients (48.9\%) have overlap with IBS and 88 of the 157 IBS patients (56.1\%) have overlap with FD. were expressed as $t$ scores with a population mean of 50 and a standard deviation of 10 .

\section{Statistical Methods}

Continuous data were presented as means $\pm \mathrm{SD}$, and categorical data were presented as proportions. For categorical data, group differences were tested using the chi-square test. The 2 -sample independent $t$ test was used for the analysis of continuous variables. A $P$-value of less than 0.05 was considered statistically significant. All statistical analyses were performed with SAS for Windows software (version 8.02, SAS Institute Inc, Cary, NC, USA).

\section{Results}

A total of 786 patients (primary clinics, 345; tertiary hospitals, 441) was participated in the present study. Among them, 391 patients $(49.7 \%)$, who fulfilled the questionnaire completely and did not have abnormal clinical examinations, were analyzed in the present study (Table 1). In the primary clinics, the FGIDs were diagnosed in 155 patients (44.9\%), consisting of 38 males and 117 females. In the tertiary hospitals, FGIDs were diagnosed in 236 patients (53.5\%), 82 males and 154 females. Average age was younger in the patients of the primary clinics than the tertiary hospitals ( $38.4 \pm 13.6$ and $44.5 \pm 15.3$ years, respectively; $P<$ $0.005)$. Gender and age distribution of the patients are shown in Table 1.

\section{Spectra of the Functional Gastrointestinal Disorders}

Spectra of the FGIDs are presented in Table 2. In all patients with the functional disorders, frequency of the esophageal, gastroduodenal, bowel, and anorectal disorders was $27.9 \%, 61.6 \%$, $66.0 \%$ and $16.9 \%$, respectively. The most prevalent FGID was

Table 1. Gender and Age Distribution of the Patients With Functional Gastrointestinal Disorders

\begin{tabular}{|c|c|c|c|}
\hline \multirow[b]{2}{*}{ Age group (yr) } & \multicolumn{2}{|c|}{ Gender } & \multirow{2}{*}{$\begin{array}{c}\text { Total } \\
(\mathrm{N}=391)\end{array}$} \\
\hline & $\begin{array}{c}\text { Male } \\
(\mathrm{n}=120)\end{array}$ & $\begin{array}{c}\text { Female } \\
(\mathrm{n}=271)\end{array}$ & \\
\hline $18-29$ & $23(19.2 \%)$ & $80(29.5 \%)$ & $103(26.3 \%)$ \\
\hline $30-39$ & $34(28.3 \%)$ & $42(15.5 \%)$ & $76(19.4 \%)$ \\
\hline $40-49$ & $29(24.2 \%)$ & $58(21.4 \%)$ & $87(22.3 \%)$ \\
\hline $50-59$ & $18(15 \%)$ & $49(18.1 \%)$ & $67(17.1 \%)$ \\
\hline$\geq 60$ & $16(13.3 \%)$ & $42(15.5 \%)$ & $58(14.8 \%)$ \\
\hline
\end{tabular}


Table 2. Frequencies of All Functional Gastrointestinal Disorders

\begin{tabular}{llccc}
\hline & & $\begin{array}{c}\text { Primary clinics } \\
(\mathrm{n}=155)\end{array}$ & $\begin{array}{c}\text { Tertiary hospitals } \\
(\mathrm{n}=236)\end{array}$ & $\begin{array}{c}\text { Total } \\
(\mathrm{N}=391)\end{array}$ \\
\hline Functional esophageal disorders & Functional heartburn & $20(12.9 \%)$ & $37(15.7 \%)$ & $57(14.6 \%)$ \\
& Functional chest pain & $10(6.5 \%)$ & $19(8.1 \%)$ & $29(7.4 \%)$ \\
& Functional dysphagia & $10(6.5 \%)$ & $12(5.1 \%)$ & $22(5.6 \%)$ \\
& Globus & $4(2.6 \%)$ & $6(2.5 \%)$ & $10(2.6 \%)$ \\
Functional gastroduodenal disorders & Functional dyspepsia & $72(46.5 \%)$ & $108(45.8 \%)$ & $180(46.0 \%)$ \\
& Belching disorders & $44(28.4 \%)$ & $76(32.2 \%)$ & $120(30.7 \%)$ \\
& Nausea and vomiting disorders & $21(13.5 \%)$ & $27(11.4 \%)$ & $48(12.3 \%)$ \\
& Rumination syndrome & $4(2.6 \%)$ & $7(3.0 \%)$ & $11(2.8 \%)$ \\
Functional bowel disorders & Irritable bowel syndrome & $70(45.2 \%)$ & $87(36.9 \%)$ & $157(40.2 \%)$ \\
& Functional bloating & $23(14.8 \%)$ & $29(12.3 \%)$ & $52(13.3 \%)$ \\
& Functional constipation & $12(7.7 \%)$ & $30(12.7 \%)$ & $42(10.7 \%)$ \\
& Functional diarrhea & $3(1.9 \%)$ & $10(4.2 \%)$ & $13(3.3 \%)$ \\
Functional anorectal disorders & Anorectal pain & $15(9.7 \%)$ & $29(12.3 \%)$ & $44(11.3 \%)$ \\
& Functional defecation disorder & $5(3.2 \%)$ & $19(8.1 \%)$ & $24(6.1 \%)$ \\
\hline
\end{tabular}

Values are number of patients, with percentages from each group in parentheses.

Table 3. Subtypes of Functional Dyspepsia

\begin{tabular}{lccc}
\hline & $\begin{array}{c}\text { Primary clinics } \\
(\mathrm{n}=72)\end{array}$ & $\begin{array}{c}\text { Tertiary hospitals } \\
(\mathrm{n}=108)\end{array}$ & $\begin{array}{c}\text { Total } \\
(\mathrm{n}=180)\end{array}$ \\
\hline PDS & $54(75.0 \%)$ & $80(74.1 \%)$ & $134(74.4 \%)$ \\
EPS & $2(2.8 \%)$ & $7(6.5 \%)$ & $9(5.0 \%)$ \\
PDS + EPS & $2(2.8 \%)$ & $2(1.9 \%)$ & $4(2.2 \%)$ \\
Neither PDS nor EPS & $14(19.4 \%)$ & $19(17.6 \%)$ & $33(18.3 \%)$ \\
\hline
\end{tabular}

PDS, postprandial distress syndrome; EPS, epigastric pain syndrome.

FD (46\%), followed by IBS (40.2\%) and belching disorders $(30.7 \%)$. In the functional esophageal disorders, functional heartburn was observed the most frequently both in the primary care clinics and tertiary care hospitals. In functional gastroduodenal disorders, FD was the most prevalent, and then followed by belching disorders, nausea and vomiting disorders (12.3\%) and rumination syndrome $(2.8 \%)$. In functional bowel disorders, IBS was the most prevalent, followed by functional bloating (13.3\%), functional constipation $(10.7 \%)$ and functional diarrhea $(3.3 \%)$. In functional anorectal disorders, frequency of anorectal pain and functional defecation disorder was $11.3 \%$ and $6.1 \%$, respectively. There were no statistical differences in frequencies between the primary clinics and the tertiary care hospitals in each of the FGIDs.

In subtypes of $\mathrm{FD}$, PDS was observed most frequently (Table 3). There was no difference in the prevalence of FD subtypes between the primary care clinics and tertiary care hospitals. Thirty-three patients (18.3\%) with FD strictly meet neither PDS
Table 4. Subtypes of Irritable Bowel Syndrome

\begin{tabular}{lccc}
\hline & $\begin{array}{c}\text { Primary clinics } \\
(\mathrm{n}=70)\end{array}$ & $\begin{array}{c}\text { Tertiary hospitals } \\
(\mathrm{n}=87)\end{array}$ & $\begin{array}{c}\text { Total } \\
(\mathrm{n}=157)\end{array}$ \\
\hline IBS-C & $9(12.5 \%)$ & $17(15.7 \%)$ & $26(16.6 \%)$ \\
IBS-D & $22(30.6 \%)$ & $24(22.2 \%)$ & $46(29.3 \%)$ \\
IBS-M & $35(48.6 \%)$ & $42(38.9 \%)$ & $77(49.0 \%)$ \\
IBS-U & $4(5.6 \%)$ & $4(3.7 \%)$ & $8(5.1 \%)$ \\
\hline
\end{tabular}

IBS-C, IBS with constipation; IBS-D, IBS with diarrhea; IBS-M, mixed IBS; IBS-U, unsubtyped IBS.

nor EPS criteria. Despite having epigastric pain or burning in the middle abdomen, the reasons that patients did not satisfy EPS criteria were due to the followings; (1) pain with less than moderate severity ( $\mathrm{n}=9$ ), (2) pain or burning without complete disappearance in the same day $(\mathrm{n}=10)$, (3) chest pain with a frequency of more than a month $(n=9),(4)$ heartburn with a frequency of more than a month $(n=2)$ and $(5)$ experience on the relief of discomfort with defecation $(n=3)$.

Subtypes of IBS are shown in Table 4. In IBS subtypes, mixed IBS (IBS-M) was most common followed by IBS with diarrhea (IBS-D) and IBS with constipation (IBS-C). There were no statistical differences in the frequency of IBS and each IBS subtype between the primary clinics and the tertiary hospitals.

IBS subclassification using the Rome III questionnaire and the Bristol stool form scale questions are shown in Table 5. The agreement was low between the 2 methods in defining IBS sub- 
Table 5. Comparison of Irritable Bowel Syndrome Subtypes Between the Rome III Questionnaire and the Bristol Stool Form Scale

\begin{tabular}{|c|c|c|c|c|c|}
\hline & & \multicolumn{4}{|c|}{ Subtypes using Bristol Stool Form Scale } \\
\hline & & $\begin{array}{c}\text { IBS-C } \\
(\mathrm{n}=38,24.2 \%)\end{array}$ & $\begin{array}{c}\text { IBS-D } \\
(\mathrm{n}=43,27.4 \%)\end{array}$ & $\begin{array}{c}\text { IBS-M } \\
(\mathrm{n}=7,4.6 \%)\end{array}$ & $\begin{array}{c}\text { IBS-U } \\
(\mathrm{n}=69,43.9 \%)\end{array}$ \\
\hline \multirow[t]{4}{*}{ Subtypes using Rome III questionnaire } & IBS-C $(n=26,16.6 \%)$ & 7 & 1 & 0 & 18 \\
\hline & IBS-D $(n=46,29.3 \%)$ & 4 & 19 & 3 & 20 \\
\hline & IBS-M $(\mathrm{n}=77,49.0 \%)$ & 25 & 23 & 4 & 25 \\
\hline & IBS-U $(\mathrm{n}=8,5.1 \%)$ & 2 & 0 & 0 & 6 \\
\hline
\end{tabular}

Abbreviations as in Table 4.

Table 6. Overlap of the Functional Gastrointestinal Disorders

\begin{tabular}{lcrc}
\hline & Primary clinics $(\mathrm{n}=155)$ & Tertiary hospitals $(\mathrm{n}=236)$ & Total $(\mathrm{N}=391)$ \\
\hline Combined FGIDs & $84(54.2 \%)$ & $117(49.6 \%)$ & $201(51.4 \%)$ \\
FD + IBS & $35(22.6 \%)$ & $53(22.5 \%)$ & $88(22.5 \%)$ \\
FD + Belching disorders & $25(16.1 \%)$ & $45(19.1 \%)$ & $70(17.9 \%)$ \\
IBS + Belching disorders & $20(12.9 \%)$ & $30(12.7 \%)$ & $50(12.8 \%)$ \\
FD + Functional heartburn & $16(10.3 \%)$ & $24(10.2 \%)$ & $40(10.2 \%)$ \\
IBS + Functional heartburn & $5(3.2 \%)$ & $15(6.4 \%)$ & $20(5.1 \%)$ \\
Functional heartburn + Belching disorders & $10(6.5 \%)$ & $19(8.1 \%)$ & $29(7.4 \%)$ \\
FD + IBS + Belching disorders & $14(9.0 \%)$ & $24(10.2 \%)$ & $38(9.7 \%)$ \\
FD + IBS + Functional heartburn & $5(3.2 \%)$ & $14(5.9 \%)$ & $19(4.9 \%)$ \\
FD + Belching disorders + Functional heartburn & $8(5.2 \%)$ & $13(5.5 \%)$ & $21(5.4 \%)$ \\
IBS + Belching disorders + Functional heartburn & $4(2.6 \%)$ & $9(3.8 \%)$ & $13(3.3 \%)$ \\
FD + IBS + Belching disorders + Functional heartburn & $4(2.6 \%)$ & $9(3.8 \%)$ & $13(3.3 \%)$ \\
\hline
\end{tabular}

FGIDs, functional gastrointestinal disorders; FD, functional dyspepsia; IBS, irritable bowel syndrome.

types (kappa value, 0.08). Seven (26.9\%) from 26 IBS-C patients responded as Bristol types 1 and 2. Nineteen (41.3\%) from 46 IBS-D patients responded as types 6 and 7 in subtype classification using the Bristol Stool Form Scale. Thereby, unsubtyped IBS (IBS-U) was unexpectedly the common subtype by stool form, whereas IBS-U was uncommon when evaluated with 2 questions of Rome III questionnaire. When the Bristol types were used, 69 from 157 IBS patients (43.9\%) were IBS-U. Among 26 patients who were subtyped as IBS-C by the Rome III questionnaire, 19 patients were not classified into IBS-C by the Bristol Stool Form Scale. In IBS-D, 27 from 46 patients were not in agreement between the 2 subtyping methods.

\section{Overlap of Functional Gastrointestinal Disorders}

Among a total of 391 patients with FGIDs, 201 patients (51\%) had more than one FGID (Table 6). The most prevalent overlap FGID was the combination of FD and IBS (22.5\%). Figure shows the overlapping of FD and IBS. In FD, the most frequent FGID overlapping with FD was IBS (48.9\%), followed by belching disorders (17.9\%) and functional heartburn (10.2\%). In IBS, the most frequent FGID overlapping with IBS was FD $(56.1 \%)$, followed by belching disorders $(12.8 \%)$ and functional heartburn $(5.1 \%)$.

Among the patients with belching disorders $(\mathrm{n}=120), 22$ patients (18\%) had only these disorders, and the others had other coexisting FGIDs, among whom the most patients had more than one FGID (55\%).

\section{Psychosomatic Symptoms}

When we compared the score of somatization, depression and anxiety between the patients with single FGID and with overlap FGIDs, every score of somatization, depression and anxiety was significantly higher in patients with overlap FGIDs (Table 7). According to gender, female patients have significantly higher score of somatization, depression and anxiety than male patients, respectively. 
Table 7. Psychologic Distress in Patients With Functional Gastrointestinal Disorders

\begin{tabular}{lcccccc}
\hline & \multicolumn{2}{c}{ Overlapping FGID } & & \multicolumn{3}{c}{ Gender } \\
\cline { 2 - 3 } & $\begin{array}{c}\text { Single FGID } \\
(\mathrm{n}=190)\end{array}$ & $\begin{array}{c}\text { Overlap FGID } \\
(\mathrm{n}=201)\end{array}$ & & $\begin{array}{c}\text { Male } \\
(\mathrm{n}=120)\end{array}$ & $\begin{array}{c}\text { Female } \\
(\mathrm{n}=271)\end{array}$ & Total $(\mathrm{N}=391)$ \\
\hline Somatization & $50.4 \pm 10.2$ & $54.0 \pm 11.5^{\mathrm{a}}$ & & $48.1 \pm 9.8$ & $54.1 \pm 11.1^{\mathrm{b}}$ & $52.2 \pm 11.1$ \\
Depression & $47.2 \pm 11.5$ & $51.0 \pm 12.8^{\mathrm{a}}$ & & $44.6 \pm 8.9$ & $51.2 \pm 13.0^{\mathrm{b}}$ & $49.1 \pm 12.3$ \\
Anxiety & $47.8 \pm 11.4$ & $52.0 \pm 13.5^{\mathrm{a}}$ & & $46.1 \pm 10.1$ & $51.7 \pm 13.3^{\mathrm{b}}$ & $50.0 \pm 12.6$ \\
\hline
\end{tabular}

FGID, functional gastrointestinal disorder.

${ }^{\mathrm{a}} P<0.005$ for comparing the overall score of patients with single FGID, ${ }^{\mathrm{b}} P<0.001$ for comparing the overall score of male patients.

\section{Discussion}

In the present study, half of the new patients who visited the primary care clinics and tertiary care hospitals had the FGIDs. Rome III questionnaire was useful in defining the FGID patients into each functional disorder in Korea. The most common FGID was FD, followed by IBS. FD was the most common both in primary care clinics and tertiary care hospitals. PDS was the most common subtype. There were few responders for constipation as Bristol types 1 and 2 and for diarrhea as types 6 and 7 in subtype classification using the Bristol Stool Form Scale. Half of the patients with FGID had multiple disorders, among whom the overlap of FD and IBS was most commonly found. Patients with more than one FGID showed higher score of psychosomatic symptoms than those with single FGID. Comparing with male FGID patients, female patients had higher score of psychosomatic symptoms.

Rome III criteria changed the diagnosis of FD to be more clarified. These were expected to restrict dyspepsia patients being diagnosed as FD and to decrease the number of FD patients compared to the Rome II criteria. However, FD was most commonly found both in the primary care clinics and tertiary care hospitals, which was the same result of our previous study using Rome II criteria. ${ }^{5}$

Belching disorders has been considered rare, although epidemiological studies remain to be defined. An Asian study reported only $1 \%$ of aerophagia from the patients with functional bowel disorder in a survey of consecutive new patients. ${ }^{6}$ In a Western study, a prevalence of $6 \%$ was reported. ${ }^{7}$ However, our study showed that $30 \%$ of total patients had belching disorders and most of the patients with these disorders (82\%) had also other FGIDs. This might arise from the relatively wide diagnostic criteria for unspecified excessive belching.
Our study revealed half of the patients to have overlap FGIDs. This is consistent with the previous studies. ${ }^{8,9}$ The individual disorders were expected to have homogeneity and more pathophysiologically relevant symptoms when these were diagnosed and classified by Rome III criteria. However, a substantial number of patients had overlap of FGIDs such as FD with IBS or functional heartburn in our results. Overlap of FGID in Rome III criteria may cause methodological pitfall in clinical research, leading to the biased conclusions and the inappropriate application of research results. FD patients with heartburn had different responsiveness to acid suppressive treatment compared with the patients without heartburn symptom. ${ }^{10-12}$ As shown in our study, this problem was not resolved completely by the Rome III criteria. Overlap FGID strengthened the importance of thorough assessment of other FGIDs when a patient was diagnosed with a functional disorder. Patients with overlap FGID may not be satisfied with treatment of a single disorder. Therefore, overlap of FGIDs needs to be carefully considered both in the clinical practice and experimental trials. A substantial body of pathophysiological researches will be needed to define the individual entities.

As shown in the previous studies, ${ }^{13,14}$ our study using Rome III criteria showed that the most common overlap FGIDs were FD and IBS, and that about half of FD patients had also IBS. To explain this finding, common pathophysiological mechanism such as visceral hypersensitivity has been assessed in the previous studies. ${ }^{15,16}$ Because visceral hypersensitivity has been shown in a subset of patients with FGID, ${ }^{17,18}$ the exact underlying mechanism for this overlapping FGIDs remains to be established.

Generally, psychologic distress is directly correlated with the severity of FGID symptoms and the degree of impairment. ${ }^{19}$ When we compared the score of somatization, depression and anxiety between the patients with single FGID and with overlap FGIDs, all scores were significantly higher in the latter patients. 
There has been no data whether overlap FGIDs are associated largely with psychologic distress. In a study, there is greater impairment of health-related quality of life and poorer outcome when chronic fatigue syndrome and IBS occurs together. ${ }^{20}$

Major change of Rome III criteria was subclassification of FD and this needs validation. In our study, frequency of EPS was interestingly low both in the primary care clinics and tertiary care hospitals, which was contradictory compared to our previous study using Rome II criteria. ${ }^{5}$ The reasons for this finding, though not easily explicable, can be speculated as follows. First, the diagnostic criteria of EPS might be too strict, such as threshold of pain severity, complete disappearance of symptoms in the same day, and no allowance for overlap with chest pain. Second, low prevalence of EPS could be influenced by the difficulty in understanding the meanings between heartburn and epigastric burning in Korean language. The order effect that questions for the esophageal disorders located ahead of the gastroduodenal disorders might have also influenced the results. More evaluation of the pathophysiology and factor analysis may be needed to clarify these findings.

There were mismatched results between the two methods for IBS subclassification. The reasons for this finding could be as follows. First, there were few responders of Bristol types 1 and 2 for constipation and types 6 and 7 for diarrhea in IBS subclassification. Thereby, IBS-U (43.9\%) was unexpectedly the most common subtype by stool form, whereas IBS-U (5.1\%) was the most uncommon by questionnaire. We found that some Korean patients considered Bristol type 3 as hard stool or type 5 as loose stool which attribute to discrepant results by the two methods. Second, the validity in the use of the Bristol Stool Form Scale, especially the cutoff value of at least $25 \%$ of bowel movements, is unknown and clearer definition is needed. Third, some patients concerned about the stability of stool form over time. Moreover, some patients might regard Bristol type 3 as hard stool or type 5 as loose stool, which could contribute to discrepant results. This finding should be considered in further validation.

Strength of the present study was as follows. First, all patients were assessed with clinical examination, laboratory tests, and upper endoscopy to rule out organic diseases. Second, new patients were enrolled consecutively both in the primary care clinics and tertiary care hospitals.

Objective tests are required in order to diagnose some functional disorders according to the Rome III criteria. However, 24 hour esophageal $\mathrm{pH}$ monitoring and anorectal function tests were not routinely performed to diagnose functional heartburn and defecation disorders, respectively, in this study. Belching disorders should also be diagnosed on objective tests. Thus, these factors should be considered when interpreting the results of the present study.

In conclusion, Rome III questionnaire was useful in defining the FGID patients into each functional disorder in Korea. Overlap FGIDs is still common by the Rome III criteria, which may limit its wide application. There were some limitations to adapt the Rome III criteria fully.

\section{Acknowledgements}

We thank to Chang Hoon Jang, Je Hyun Shin and Suk Joon Park for participating in our study and collecting the data in the primary clinics.

\section{References}

1. Drossman DA. The functional gastrointestinal disorders and the Rome II process. Gut 1999;45(suppl 2):II1-II5.

2. Drossman DA. The functional gastrointestinal disorders and the Rome III process. Gastroenterology 2006;130:1377-1390.

3. Derogatis LR. SCL-90-R: administration, scoring and procedure manual. Baltimore: Clinical Psychometrics Research 1983:55-58.

4. Kim KI, Kim JH. The standardization study of symptom checklist-90-revision in Korea III. Korean J Mental Health Res 1984;2: 278-311.

5. Choi H, Choi MG, Kim SW, et al. Functional gastrointestinal disorders in patients with gastrointestinal symptoms. Korean J Gastroenterol 1999;33:741-748.

6. Kwan AC, Bao TN, Chakkaphak S, et al. Validation of Rome II criteria for functional gastrointestinal disorders by factor analysis of symptoms in Asian patient sample. J Gastroenterol Hepatol 2003; 18:796-802.

7. Tosetti C, Stanghellini V, Corinaldesi R. The Rome II Criteria for patients with functional gastroduodenal disorders. J Clin Gastroenterol 2003;37:92-93.

8. Talley NJ, Dennis EH, Schettler-Duncan VA, Lacy BE, Olden KW, Crowell MD. Overlapping upper and lower gastrointestinal symptoms in irritable bowel syndrome patients with constipation or diarrhea. Am J Gastroenterol 2003;98:2454-2459.

9. Agréus L, Svärdsudd K, Nyrén O, Tibblin G. Irritable bowel syndrome and dyspepsia in the general population: overlap and lack of stability over time. Gastroenterology 1995;109:671-680.

10. Noh YW, Jung HK, Kim SE, Jung SA. Overlap of erosive and non-erosive reflux diseases with functional gastrointestinal disorders according to Rome III criteria. J Neurogastroenterol Motil 2010; 16:148-156.

11. Holtmann G, Talley NJ, Liebregts T, Adam B, Parow C. A placebo-controlled trial of itopride in functional dyspepsia. $\mathrm{N}$ Engl J Med 2006;354:832-840.

12. Talley NJ, Tack J, Ptak T, Gupta R, Giguère M. Itopride in func- 
tional dyspepsia: results of two phase III multicentre, randomised, double-blind, placebo-controlled trials. Gut 2008;57:740-746.

13. Corsetti M, Caenepeel P, Fischler B, Janssens J, Tack J. Impact of coexisting irritable bowel syndrome on symptoms and pathophysiological mechanisms in functional dyspepsia. Am J Gastroenterol 2004;99:1152-1159.

14. Wang A, Liao X, Xiong L, et al. The clinical overlap between functional dyspepsia and irritable bowel syndrome based on Rome III criteria. BMC Gastroenterol 2008;8:43.

15. Trimble KC, Farouk R, Pryde A, Douglas S, Heading RC. Heightened visceral sensation in functional gastrointestinal disease is not site-specific. Evidence for a generalized disorder of gut sensitivity. Dig Dis Sci 1995;40:1607-1613.

16. Holtmann G, Goebell H, Talley NJ. Functional dyspepsia and irritable bowel syndrome: is there a common pathophysiological basis? Am
J Gastroenterol 1997;92:954-959.

17. Tack J, Caenepeel P, Fischler B, Piessevaux H, Janssens J. Symptoms associated with hypersensitivity to gastric distention in functional dyspepsia. Gastroenterology 2001;121:526-535.

18. Coffin B, Azpiroz F, Guarner F, Malagelada JR. Selective gastric hypersensitivity and reflex hyporeactivity in functional dyspepsia. Gastroenterology 1994;107:1345-1351.

19. Katon W, Lin E, Von Korff M, Russo J, Lipscomb P, Bush T. Somatization: a spectrum of severity. Am J Psychiatry 1991;148: 34-40.

20. Sperber AD, Carmel S, Atzmon Y, et al. Use of the Functional Bowel Disorder Severity Index (FBDSI) in a study of patients with the irritable bowel syndrome and fibromyalgia. Am J Gastroenterol 2000;95:995-998. 